\title{
O impacto demográfico e seus diferenciais por sexo nos custos assistenciais da saúde suplementar no Brasil
}

\section{The demographic impact and its differentials by gender on the health care costs of supplementary health in Brazil}

\author{
Lucilvo Flávio dos Santos Borba Filho' (D), Pamila Cristina Lima Siviero² (D), \\ Luana Junqueira Dias Myrrha ${ }^{3 *}$ (D)
}

'Ciências Atuariais, Universidade Federal do Rio Grande Do Norte (UFRN) - Natal (RN), Brasil.

2Demografia, Departamento de Ciências Atuariais da Escola Paulista de Política, Economia e Negócios, Universidade Federal de São Paulo (UNIFESP/EPPEN) - São Paulo (SP), Brasil.

${ }^{3}$ Demografia, Departamento de Demografia e Ciências Atuariais da Universidade Federal do Rio Grande do Norte (UFRN) -

Parnamirim (RN), Brasil.

Como citar: Borba Filho LFS, Siviero PCL, Myrrha LJD. O impacto demográfico e seus diferenciais por sexo nos custos assistenciais da saúde suplementar no Brasil. Cad Saúde Colet, 2021;29(esp.):28-39. https://doi.org/10.1590/1414$462 \times 202199010299$.

\section{Resumo}

Introdução: As consequências do processo de Transição Demográfica afetam a demanda por saúde e, por conseguinte, o setor de saúde suplementar. Objetivo: Examinar as consequências futuras do envelhecimento populacional e dos ganhos em longevidade, diferenciados por sexo, nos custos assistenciais das operadoras de planos de saúde. Para tanto, utilizaram-se os dados disponibilizados pela Agência Nacional de Saúde Suplementar (ANS) e pelo Instituto Brasileiro de Geografia e Estatística (IBGE). Método: Utilizou-se o modelo de padronização, entre 2016 e 2045, variando apenas a estrutura etária populacional. Resultados: Os resultados indicam que o processo de envelhecimento populacional é mais acentuado para a população feminina e mais intenso entre os beneficiários dos planos individuais/ familiares se comparados aos dos planos coletivos. As internações e os exames complementares permanecerão os mais onerosos entre os itens assistenciais. Em relação aos impactos da longevidade, constatou-se que os maiores gastos esperados de usuários com mais de 59 anos de idade até a morte são com as internações e entre as mulheres, nos planos individuais/familiares. Conclusão: $O$ envelhecimento populacional e aumento de longevidade, principalmente para mulheres, vão impactar os custos dos planos de saúde, com maior intensidade os individuais/familiares. A saúde suplementar precisa se preparar para os desafios demográficos impostos aos custos futuros, buscando políticas capazes de minimizá-los.

Palavras-chave: dinâmica populacional; saúde suplementar; previsões demográficas; Brasil.

\section{Abstract}

Background: The consequences of the Demographic Transition process affect the demand for health, including the private health sector. Objective: To examine the consequences of population aging and the longevity gains, gender differentials, in the healthcare costs of healthcare operators. Data from The National Regulatory Agency for Private Health Insurance and Plans (Agência Nacional de Saúde Suplementar (ANS)) and the Brazilian Institute of Geography and Statistics (Instituto Brasileiro de Geografia e Estatística (IBGE)) were used for this purpose. Method: The standardization model has been used between 2016 and 2045, varying only the population age structure. Results: The results indicate that the population

Trabalho realizado na Universidade Federal do Rio Grande do Norte (UFRN) - Natal (RN), Brasil.

Correspondência: luanamyrrha@gmail.com.

Fonte de financiamento: nenhuma.

Conflito de interesses: nada a declarar.

Recebido em: Ago. 14, 2019. Aprovado em: Jan. 02, 2020
Este é um artigo publicado em acesso aberto (Open Access) sob a licença Creative Commons Attribution, que permite uso, distribuição e reprodução em qualquer meio, sem restrições desde que o trabalho original seja corretamente citado. 
aging process is more accentuated for the female population and more intense among the beneficiaries of individual/family plans compared to collective plans. The hospitalization and complementary examinations showed the highest absolute monetary values between care items, for both types of plans, in all years analyzed. The findings of the impacts of longevity, the expected costs for the users of the last age group will be higher for hospitalizations, especially among women and with individual / family plans. Conclusion: Population aging and increased longevity, especially for women, will impact the costs of health plans, with greater intensity for individual / family members. Supplementary health needs to prepare for the demographic challenges imposed by future costs by pursuing policies that can minimize them.

Keywords: population dynamics; supplemental health; population forecast; Brazil.

\section{INTRODUÇÃO}

A redução nos níveis de mortalidade e fecundidade, processo conhecido como transição demográfica, vem ocorrendo na maioria dos países, incluindo o Brasil, e tem como consequência a mudança na composição por idade e sexo da população, com a redução dos grupos etários mais novos e o alargamento dos grupos etários mais velhos ${ }^{1}$.

A diminuição da fração jovem na população é um processo ocasionado principalmente pela queda da fecundidade, que durante uma janela do tempo proporciona o aumento relativo das pessoas em idade ativa, o que pode contribuir para o crescimento da poupança e da produtividade. Posteriormente, observa-se o aumento da proporção das pessoas idosas, que consumirão a poupança construída durante a idade ativa, acarretando efeitos na poupança privada e nos gastos públicos com saúde e pensões².

Nesse sentido, torna-se necessária maior atenção para uma das mais importantes consequências desse processo de envelhecimento populacional: as demandas de saúde. O aumento da proporção de idosos na população seria capaz de produzir um crescimento significativo nos custos relativos de grupamentos de doenças mais predominantes nas idades avançadas, como neoplasias e doenças circulatórias ${ }^{3}$. No Brasil, os custos de saúde para pessoas idosas são altos, sendo observado um aumento gradativo na taxa de internação a partir da quinta década de vida, e na faixa de 80 anos ou mais essa taxa quadruplica quando comparada à dos 50 anos de idade 4 .

Paralelamente ao processo de envelhecimento da população, observam-se quedas ininterruptas nos níveis de mortalidade das populações e, consequentemente, mudanças nos padrões de causas de óbito. Essas mudanças têm levado ao aumento da longevidade e também produzido alguns diferenciais, como aquele observado entre os sexos ${ }^{5}$. O diferencial por sexo na sobrevivência frequentemente supera outros diferenciais, tais como local de residência (urbano vs rural), etnia e status socioeconômicos ${ }^{6}$. Na grande maioria dos países, a mortalidade masculina é maior do que a feminina, assim como a esperança de vida ao nascer e em outras idades é maior para as mulheres ${ }^{78}$.

Razões biológicas e não biológicas vêm sendo arroladas para explicar essas diferenças entre homens e mulheres. Estudos apontam que a maior parte do diferencial é atribuída a fatores não biológicos ${ }^{910} 11$, entre os quais se destacam os comportamentos mais arriscados dos homens em relação ao estilo de vida e o maior cuidado das mulheres com a própria saúde ${ }^{12} 13$.

O sistema de saúde no Brasil é heterogêneo, e duas formas de intermediação convivem paralelamente: a pública, representada pelo Sistema Único de Saúde (SUS), e a privada ou suplementar, que é composta pelas operadoras de planos de saúde. Tanto o SUS quanto as operadoras de planos de saúde utilizam uma mesma rede de prestadores de serviços, hegemonicamente privada, para atender às demandas de serviços médicos ${ }^{14}$.

A saúde suplementar exerce um papel fundamental na demanda dos serviços assistenciais no Brasil, apresentando altos graus de satisfação entre seus usuários, algo pouco frequente no sistema público. Ademais, caracteriza-se como um pilar essencial na sustentação do sistema de saúde brasileiro, sendo indispensável para o Estado, pois este não teria como arcar com a integração dos gastos do setor privado no orçamento da União ${ }^{15}$.

O objetivo deste trabalho é analisar os impactos do envelhecimento populacional, do aumento na expectativa de vida e seus diferenciais por sexo nos gastos futuros dos planos 
de saúde privados. Espera-se que, no médio prazo, o sistema privado de saúde brasileiro se depare com altos custos assistenciais, provenientes de uma expressiva massa de beneficiários idosos. Essa parcela da população, que já demanda mais serviços de saúde, deve ampliar a permanência em exposição a possíveis cuidados, resultante dos ganhos em longevidade.

\section{MÉTODO}

A fonte de dados principal deste estudo é proveniente da Agência Nacional de Saúde Suplementar (ANS), disponíveis no ANS Tabnet. A ANS Tabnet permite realizar várias consultas de dados sobre os beneficiários, planos privados de saúde, operadoras, demandas do consumidor e ressarcimento ao SUS. As informações estão disponíveis a partir de março de 2000, com intervalos trimestrais de atualizações. Vale ressaltar que os dados referentes à mortalidade só estão disponíveis a partir do ano de 2004. Os dados coletados informam a quantidade de usuários de planos de assistência médica por sexo e tipo de contratação (individual/familiar e empresarial/coletivo/adesão), distribuídos por faixas etárias de reajuste e faixas etárias quinquenais.

Para estimar a população futura de beneficiários da saúde suplementar brasileira e o efeito dos ganhos em longevidade nos custos assistenciais, foi tomada como base as projeções do IBGE revisadas no ano de 2013, as quais consideram um horizonte temporal de 50 anos $^{16}$.

Quanto às informações relativas aos custos assistenciais da saúde suplementar, são utilizados os dados fornecidos pela ANS na edição de número 5 do Painel de Precificação de Planos de Saúde, provindas das Notas Técnicas de Registro de Produto (NTRP) enviadas pelas operadoras $^{17}$. As informações coletadas são referentes aos anos 2014, 2015 e 2016.

Os planos médico-hospitalares apresentam onze segmentações assistenciais, as quais são agrupadas em três:"Ambulatorial","Hospitalar", e“Ambulatorial + Hospitalar" para apresentação do Painel ${ }^{17}$.

No presente trabalho são utilizados os dados de custos por expostos, do Painel de Precificação, referentes aos planos com cobertura "Ambulatorial + Hospitalar", que cobrem os seguintes itens de despesa: consultas médicas, demais despesas assistenciais, exames complementares, internações, outros atendimentos ambulatoriais e terapias. Esses itens são apresentados como média simples, excluídos os valores extremos, desagregados entre os seguintes tipos de contratação: individual/familiar; e coletivo/empresarial/adesão.

O método utilizado neste trabalho é determinístico, de modo que o efeito demográfico sobre o total dos custos assistenciais será mensurado considerando que o custo médio por exposto do triênio 2014, 2015 e 2016 permanece constante ao longo do tempo. Nesse cenário, somente a composição etária e por sexo, juntamente com o tamanho da população, terão impacto no total dos custos assistenciais futuros.

Essa análise é denominada por Miller ${ }^{18}$ como "Modelo Etário". De acordo com Rodrigues apud Strunk ${ }^{19}$, esta metodologia facilita o momento de mensuração dos dados, pois a análise não necessita de processos avançados de cálculo. Importante ressaltar que vários outros fatores, além do demográfico, interferem nos custos assistenciais do setor privado de saúde, entre os quais se destaca a inflação do setor de saúde suplementar dada, em sua grande maioria, pela elevada inserção tecnológica de novos serviços, bens, procedimentos e práticas ${ }^{20}$. Embora o cenário escolhido seja conservador, uma vez que se espera que o custo por exposto não permaneça constante com o passar dos anos, ele é adequado para o objetivo do estudo, que é verificar apenas o impacto das mudanças demográficas nos custos assistenciais.

Para obter a projeção dos beneficiários da saúde suplementar por meio das projeções da população brasileira do IBGE ${ }^{16}$, foi necessário estimar uma taxa de cobertura dos planos de saúde que, segundo o ANS Tabnet, é a proporção da quantidade de beneficiários na população. O ANS Tabnet dispõe desta informação, porém apresenta tais dados em faixas etárias que não condizem com as dispostas nos dados sobre os custos assistenciais. No Painel de Precificação ${ }^{17}$, os valores dos custos assistenciais são distribuídos nas dez faixas etárias de reajuste e precificação dos planos de saúde, dadas pela RN № 63, de 22 de dezembro de 
$2003^{21}$. Portanto, fez-se necessário calcular a taxa de cobertura para as mesmas faixas etárias dos custos assistenciais.

Para estimar a taxa de cobertura nas faixas etárias quinquenais por sexo e tipo de contração, utilizamos a média de 3 anos subsequentes, da razão entre o número de beneficiários de assistência médica para cada tipo de contratação, por faixa etária e sexo, em todo o território nacional, e a população brasileira projetada para os anos de 2014, 2015 e 2016, conforme a Equação 1:

$$
\begin{aligned}
& T x \operatorname{Cob}_{f, s, c}^{\text {anual }}=\left[\left(\operatorname{Benef}_{f, s, c}^{2016} \div \operatorname{PopBr}_{f, s}^{2016}\right)+\left(\operatorname{Benef}_{f, s, c}^{2015} \div \operatorname{PopBr}_{f, s}^{2015}\right)+\left(\operatorname{Benef}_{f, s, c}^{2014} \div \operatorname{PopBr}_{f, s}^{2014}\right)\right] \div 3 \\
& \text { Onde: } \\
& f \text { = faixa etária; } \\
& s=\text { sexo; } \\
& c=\text { tipo de contratação; } \\
& T x C o b_{f, s, c}^{\text {anual }}=\text { Taxa média anual de cobertura dos planos privados de assistência médica, }
\end{aligned}
$$
na faixa etária $f$, do sexo $s$ e tipo de contratação $c$;

Benef $_{f, s, c}^{2016,2015 o u 2014}=$ Quantidade média de beneficiários de planos privados de assistência médica no ano de 2016/2015/2014, na faixa etária $f$, do sexo $s$ e tipo de contratação c;

$\operatorname{PopBr}_{f, s}^{2016,2015 \text { ou } 2014}=$ População brasileira no ano de 2016/2015/2014 na faixa etária $f$ e do sexo $s$.

Assim, a quantidade de beneficiários será projetada de 2016 a 2045, com intervalos de 10 anos (com exceção do primeiro intervalo, que será de 9), mantendo-se constante a taxa de cobertura de planos privados de assistência médica obtida na equação 1. Para estas projeções, utiliza-se como base a fórmula proposta por Carneiro ${ }^{20}$ (Equação 2):

$$
i_{i} \text { Benef }_{f, s, c}=T x \operatorname{Cob}_{f, s, c}^{\text {anual }} \times{ }_{i} \operatorname{PopBr}_{f, s}
$$

Onde:

$t=$ tempot $(2025,2035,2045)$;

$f$ = faixa etária;

$s=$ sexo;

$c$ = tipo de contratação;

${ }_{i}$ Benef $_{f, s, c}=$ Quantidade de beneficiários de planos privados de assistência médica no ano $t$, da faixa etária $f$, do sexo $s$ e tipo de contratação $c$;

$T x \operatorname{Cob}_{f, s, c}^{\text {anual }}=$ Taxa média anual de cobertura dos planos privados de assistência médica, na faixa etária $f$, do sexo $s$ e tipo de contratação $c$;

${ }_{i} \operatorname{PopBr}_{f, s}=$ População brasileira no ano ${ }^{t}$, da faixa etária ${ }^{f}$ e do sexo ${ }^{s}$.

Para as projeções referentes aos custos assistenciais, também é adotada a fórmula utilizada por Carneiro20, embora sejam feitos alguns ajustes para a realidade do presente estudo. Como a ANS não disponibiliza os dados sobre os custos assistenciais por sexo, assume-se que para ambos os sexos os custos são iguais e constantes para todos os anos projetados. A fórmula geral utilizada para as projeções dos custos assistenciais, para homens e mulheres, é (Equação 3):

${ }_{i} C A P_{f, c . i}=C A_{f, c, i}^{2016} \times{ }_{i}$ Benef $_{f, c}$

Onde:

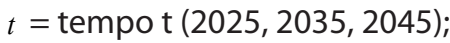

$f$ = faixa etária;

$c$ = tipo de contratação;

$i=$ item de despesa assistencial;

${ }_{i} C A P_{f, c . i}=$ Custo assistencial projetado $i$ no ano t, na faixa etária $f$, e tipo de contratação $c$; $C A_{f, c, i}^{2016}=$ Custo assistencial $i$ no ano de 2016, na faixa etária $f$, e tipo de contratação $c$; ${ }_{i}$ Benef $_{f, c}=$ Quantidade de beneficiários de planos privados de assistência médica no ano $t$, da faixa etária $f$, e tipo de contratação $c$. 
No que diz respeito ao efeito do aumento da longevidade, considera-se a evolução da expectativa de vida a partir dos 60 anos. Os custos assistenciais de 2016 são fixados para todos os anos de estimação. O resultado representará o gasto futuro esperado por cada indivíduo com 60 anos de idade até a sua morte. Para chegar a esse gasto esperado, multiplica-se o custo anual médio por exposto de uma pessoa com 60 anos, que faz parte da faixa etária 59 anos de idade ou mais (faixa etária limite estabelecida pela ANS para precificação dos planos de saúde) pela expectativa de vida aos 60 anos disponibilizadas nas projeções do IBGE ${ }^{16}$, ou seja, pelo total de anos a serem vividos por um indivíduo de 60 anos, segundo o regime de mortalidade adotado.

A fórmula geral utilizada para as estimações dos custos assistenciais com base na esperança de vida para homens e mulheres (Equação 4).

${ }_{i} C E F_{59+, c, i}=C A_{59+, c, i}^{2016} \times{ }_{i} e_{60}$

Onde:

$t=$ Tempo $\mathrm{t}(2025,2035,2045)$;

$c=$ Tipo de contratação;

$i=$ Item de despesa assistencial;

${ }_{i} C E F_{59+, c, i}=$ Custo esperado até o fim da vida com o item assistencial , para beneficiários com 59 ou mais anos de idade, no tipo de contratação $c$;

$C A_{f, c, i}^{2016}=$ Custo assistencial $i$ no ano de 2016, na faixa etária de 59 anos ou mais, e tipo de contratação $c$;

${ }_{i} e_{60}=$ Expectativa de vida da população brasileira aos 60 anos de idade, no ano $t$.

\section{RESULTADOSE DISCUSSÃO}

Segundo os dados da ANS ${ }^{22}$, para o ano de 2016, os planos coletivos de assistência médica são os mais frequentes na população brasileira, correspondendo a $80,2 \%$ dos beneficiários dos planos de saúde no país, ao passo que os planos individuais/familiares representam 19,8\% desse mercado.

A população de beneficiários de planos individuais/familiares é mais envelhecida do que a dos planos de saúde coletivos (comparação entre os Gráficos 1 e 2). $O$ topo da pirâmide etária dos planos individuais/familiares (Gráfico 1) é expressivamente mais largo do que os planos coletivos (Gráfico 2), que têm uma maior participação de pessoas adultas, entre 18 e 50 anos de idade, pois são planos oferecidos para a população que está inserida no mercado de trabalho.

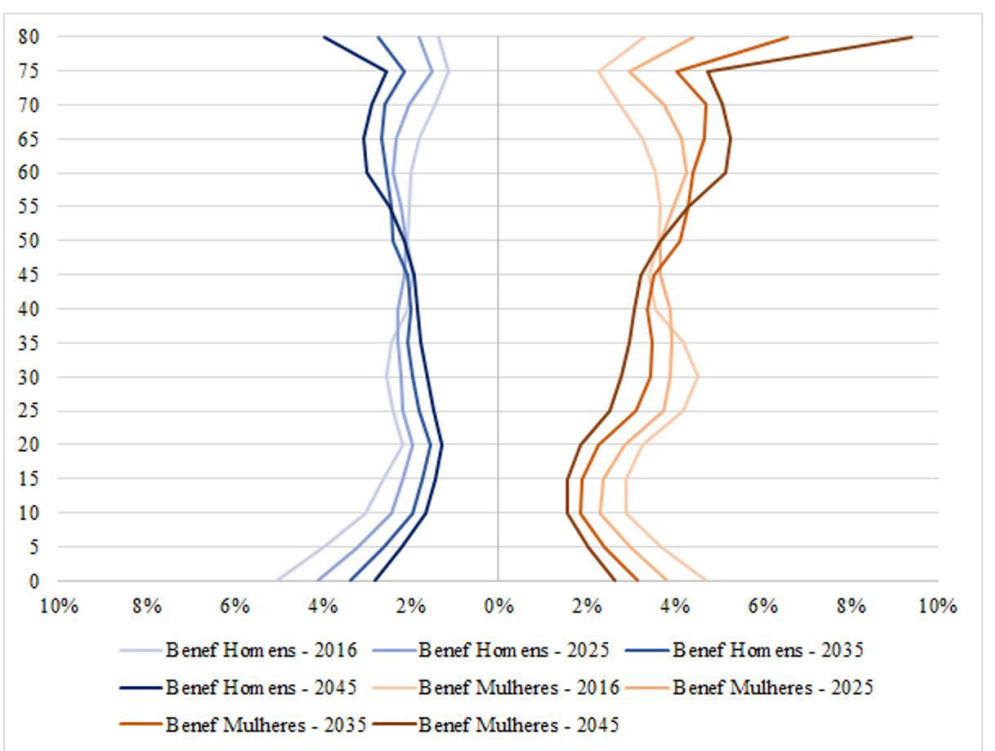

Gráfico 1. Pirâmide etária dos beneficiários de planos individuais/familiares, Brasil, 2016 a 2045. Fonte dos dados básicos: ANS 2014 a 2016, e projeções do IBGE 2013 
Com o processo de envelhecimento, percebe-se que, em ambos os grupos, o peso relativo dos idosos tende a aumentar, e o inverso ocorre para os grupos etários mais jovens. Destaca-se que o alargamento do topo é mais acentuado no sexo feminino, principalmente nos planos individuais/familiares. Em 2045, aproximadamente 10\% dos beneficiários dos planos individuais/familiares e $4 \%$ da população com planos de saúde coletivos serão mulheres com 80 anos ou mais (Gráfico 2).

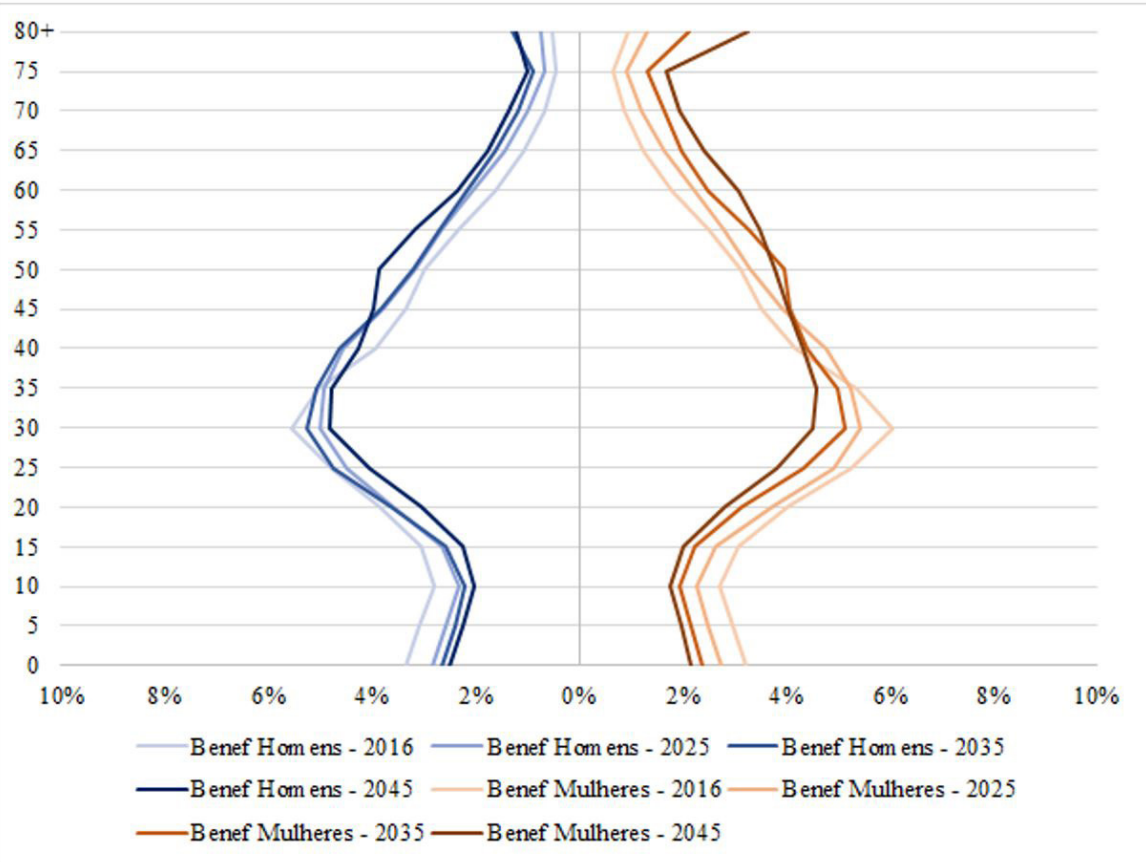

Gráfico 2. Pirâmide etária dos beneficiários de planos de planos coletivos (empresarial ou por adesão), Brasil, 2016 a 2045. Fonte dos dados básicos: ANS 2014 a 2016, e projeções do IBGE 2013

As demandas assistenciais tendem a ser mais caras e frequentes nas idades mais avançadas. Além disso, as mulheres são as que mais utilizam os serviços oferecidos pelas operadoras de saúde ${ }^{23}$. Consequentemente, o esperado é que o processo de envelhecimento populacional, em conjunto com a feminização dos usuários dos planos de saúde nas faixas etárias acima de 59 anos, contribua significativamente para o aumento dos custos da saúde suplementar no Brasil, independente da inflação do setor e das variações nos preços de cada item assistencial.

\section{Projeção do gasto com os principais itens de custo assistencial}

Para estimar o custo total futuro com cada item assistencial para os dois tipos de contratação, utilizou-se a fórmula de ${ }^{\text {Carneiro20, }}$, descrita na metodologia, para estimar o custo de cada faixa etária com o referido item e somaram-se os gastos de todas as faixas etárias com esse mesmo item. De acordo com a Tabela 1, as Internações exibiram os maiores custos absolutos entre os itens assistenciais, para os dois tipos de plano e em todos os anos estudados. Em segundo lugar estão os gastos com os Exames Complementares.

De 2016 para 2045, a expectativa é que o valor anual gasto com internações de homens em planos individuais/familiares aumente de $\mathrm{R} \$ 3,7$ bilhões para 6,6 bilhões (crescimento de $78,38 \%$ ), e para o sexo feminino, a estimativa é de que este valor passe de 7,6 bilhões para 14,4 bilhões (aumento de $89,05 \%$ ). Nos planos com tipo de contratação coletiva, empresarial ou por adesão, os gastos com internações de homens vão subir de 14,7 bilhões de para 21,2 milhões (aumento de 44,24\%). Já em relação às mulheres, espera-se um aumento de 53,19\% no período em análise (R\$ 17,8 bilhões em 2016 para R\$ 27,3 bilhões em 2045). 
Tabela 1. Custos por item de despesa assistencial, tipo de contratação e sexo, 2016-2045

\begin{tabular}{|c|c|c|c|c|c|c|c|}
\hline $\begin{array}{c}\text { Tipo de } \\
\text { Contratação }\end{array}$ & $\begin{array}{l}\text { Item de Despesa } \\
\text { Assistencial }\end{array}$ & \multicolumn{3}{|c|}{ Homens } & \multicolumn{3}{|c|}{ Mulheres } \\
\hline \multirow{6}{*}{$\begin{array}{l}\text { Individual/ } \\
\text { Familiar }\end{array}$} & Consultas médicas & $1.538,69$ & $2.067,70$ & $34,38 \%$ & $2.615,87$ & $4.011,84$ & $53,37 \%$ \\
\hline & $\begin{array}{l}\text { Demais Despesas } \\
\text { Assistenciais }\end{array}$ & 704,07 & $1.228,83$ & $74,53 \%$ & $1.418,99$ & $2.673,73$ & $88,42 \%$ \\
\hline & $\begin{array}{l}\text { Exames } \\
\text { Complementares }\end{array}$ & $1.477,00$ & $2.391,89$ & $61,94 \%$ & $2.900,77$ & $5.069,36$ & $74,76 \%$ \\
\hline & Internações & $3.777,45$ & $6.612,38$ & $75,05 \%$ & $7.637,56$ & $14.438,57$ & $89,05 \%$ \\
\hline & $\begin{array}{l}\text { Outros atendimentos } \\
\text { ambulatoriais }\end{array}$ & 390,89 & 660,08 & $68,86 \%$ & 772,03 & $1.418,25$ & $83,71 \%$ \\
\hline & Terapias & 356,36 & 643,19 & $80,49 \%$ & 737,98 & $1.413,66$ & $91,56 \%$ \\
\hline \multirow{6}{*}{$\begin{array}{l}\text { Coletivo } \\
\text { Empresarial/ } \\
\text { Adesão }\end{array}$} & Consultas médicas & $6.759,50$ & $7.876,69$ & $16,53 \%$ & $7.572,29$ & $9.276,61$ & $22,51 \%$ \\
\hline & $\begin{array}{l}\text { Demais Despesas } \\
\text { Assistenciais }\end{array}$ & $2.354,59$ & $3.389,31$ & $43,94 \%$ & $2.849,50$ & $4.353,25$ & $52,77 \%$ \\
\hline & $\begin{array}{l}\text { Exames } \\
\text { Complementares }\end{array}$ & $6.812,89$ & $9.201,63$ & $35,06 \%$ & $8.045,44$ & $11.412,62$ & $41,85 \%$ \\
\hline & Internações & $14.728,19$ & $21.243,46$ & $44,24 \%$ & $17.878,96$ & $27.389,56$ & $53,19 \%$ \\
\hline & $\begin{array}{l}\text { Outros atendimentos } \\
\text { ambulatoriais }\end{array}$ & $1.641,10$ & $2.342,77$ & $42,76 \%$ & $1.978,45$ & $2.989,25$ & $51,09 \%$ \\
\hline & Terapias & $1.401,27$ & $2.071,29$ & $47,82 \%$ & $1.706,90$ & $2.659,50$ & $55,81 \%$ \\
\hline
\end{tabular}

Fonte dos dados básicos: ANS 2014 a 2017, e projeções do IBGE 2013

Para os exames complementares dos planos individuais/familiares, os custos para os homens subiriam de R\$ 1,4 bilhão em 2016 para R\$ 2,3 bilhões em 2045 (61,94\%). Para as mulheres, espera-se que o valor de R\$ 2,9 bilhões em 2016 cresça para R\$ 5 bilhões em 2045 (74,76\%). Nos planos coletivos, os resultados das projeções apontam um crescimento de $41,85 \%$ para as mulheres ( $R \$ 11,4$ bilhões em 2045) e de 35,06\% para os homens ( $R \$ 9,2$ bilhões em 2045).

Embora nos planos individuais/familiares e coletivos os custos monetários mais elevados em 2045 serão referentes às internações e aos exames complementares, os gastos com as terapias apresentarão o maior percentual de aumento entre 2016 e 2045 , de $80,49 \%$ para os homens e $91,56 \%$ para as mulheres nos planos individuais/familiares, e $47,82 \%$ e $55,81 \%$ nos planos coletivos, respectivamente.

Os gastos por idade são maiores nos grupos etários extremos. Porém, o custo da última faixa etária, em comparativo com o primeira, é mais elevado e se mantém em todos os anos de projeção, porque esse será o grupo populacional que mais crescerá no período analisado. A título de exemplo, o Gráfico 3 apresenta os gastos com internações nos planos coletivos por grupo etário e sexo, de 2016 a 2045. Nos anos de 2016 e 2025, as nove primeiras faixas etárias, excluindo-se a de 59 anos ou mais, apresentam uma distribuição homogênea de gastos, para ambos os sexos. Nos anos de 2035 e 2045, os altos custos são visualizados mais claramente nas faixas de idade mais elevadas.

Cabe destacar que essa mesma análise foi realizada para todos os itens assistenciais e tipos de contratação. Em todos os casos, os custos variam na mesma proporção do crescimento da população. Consequentemente, em 2045, os resultados apontam para uma expressiva superioridade dos gastos da última faixa de idade, comparados aos demais grupos de idade. $E$ o gasto total com as mulheres com 59 anos ou mais de idade se manterá superior aos homens, em todos os anos e tipos de contratação. 


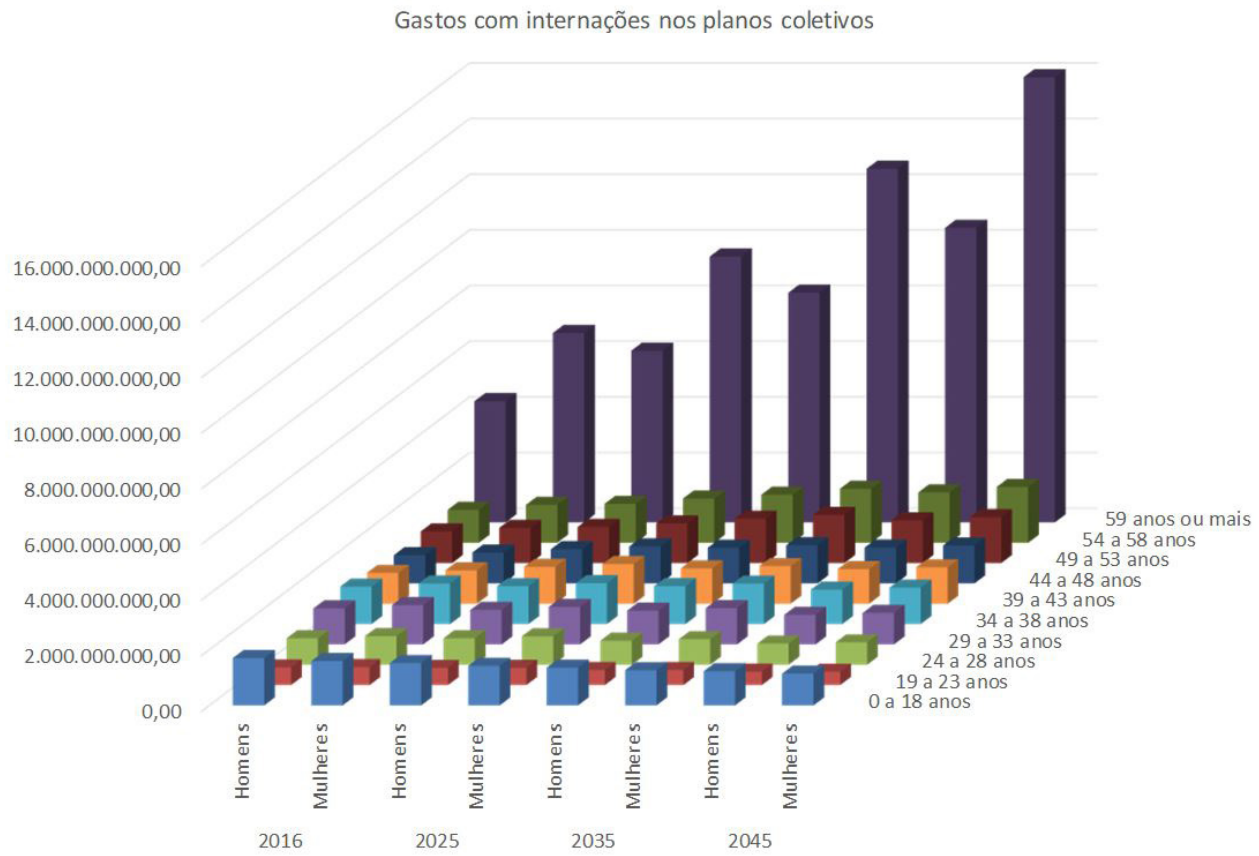

Gráfico 3. Estimativa dos gastos com internações nos planos de planos coletivos (empresarial ou por adesão), por grupo etário de precificação e sexo, Brasil, 2016 a 2045. Fonte dos dados básicos: ANS 2014 a 2017, e projeções do IBGE 2013

\section{Projeção dos custos esperados para o beneficiário até o término de sua vida}

O efeito dos ganhos em longevidade também evidencia que as internações e os exames complementares são os dois itens de custos assistenciais de maiores valores monetários no futuro, para ambos os sexos e tipos contratação. Os maiores custos esperados com internações estão no sexo feminino, e em específico nos planos individuais/familiares. Salienta-se ainda que, para toda a análise, no que diz respeito ao efeito dos ganhos em longevidade, as mulheres apresentam gastos significativamente superiores aos dos homens.

Esse custo depende da expectativa de vida e do valor médio anual gasto com um indivíduo da faixa etária de precificação 59 anos ou mais. As mulheres apresentam um tempo médio de vida a partir dos 60 anos superior ao dos homens, mas o custo médio adotado neste estudo é o mesmo para ambos os sexos. Portanto, esse efeito estimado diz respeito apenas ao aumento da longevidade das mulheres.

Em 2016, em média, o custo esperado com internações de um beneficiário de plano individual/familiar do sexo masculino com 60 anos de idade até este vir a óbito, considerando a expectativa de vida desse indivíduo a partir de 60 anos e custo anual por exposto a partir dessa idade, é de $\mathrm{R} \$ 50$ mil. Para as mulheres, essa estimativa sobe para $\mathrm{R} \$ 59 \mathrm{mil}$. Com o passar do tempo e os consequentes ganhos em longevidade, em 2045 os custos com internação masculina serão de R\$ 56 mil, e para as mulheres de R\$ 66 mil. O custo esperado até o término da vida com exames complementares em planos individuais/familiares com 60 anos de idade em 2016 é de R\$18,4 mil para mulheres e R\$15,6 mil para os homens. Em 2045, esses custos subirão para $\mathrm{R} \$ 20,5$ mil no sexo feminino e para $\mathrm{R} \$ 17,4$ mil no masculino.

Quando se trata de planos coletivos, para o ano de 2016, o custo médio esperado com internações de um beneficiário com 60 anos de idade até a sua morte é de R\$ 46 mil para os homens e de R\$ 55 mil para as mulheres. Em 2045, esses valores aumentarão para R\$ 52 mil e $\mathrm{R} \$ 61,6$ mil, respectivamente. Para exames complementares, o custo esperado de beneficiários homens em planos coletivos com 60 anos ou mais de idade é de $R \$ 15,8$ mil e para as mulheres é de $\mathrm{R} \$ 18,7$ mil. Para o ano de 2045, esses custos subirão para $\mathrm{R} \$ 20,9$ mil, quando se refere ao sexo feminino, e para $\mathrm{R} \$ 17,7$ mil para o masculino. 
Para um beneficiário acima de 60 anos, os custos esperados com internações, consultas médicas, demais despesas assistenciais e terapias são mais elevados para os planos individuais/ familiares, em todos os anos avaliados. Este cenário é invertido quando se observam os itens exames complementares e outros procedimentos ambulatoriais, nos quais os custos em planos coletivos com um beneficiário acima dos 59 anos de idade são superiores aos individuais/ familiares. A seletividade de participantes nos planos individuais/familiares é uma possível justificativa para esses resultados, uma vez que tais planos tendem a atrair pessoas que de fato vão utilizar os serviços, além do perfil etário mais idoso desse tipo de contratação. Por outro lado, os planos coletivos são destinados geralmente aos trabalhadores de empresas, conselhos ou sindicatos, que se inserem no plano independente das necessidades. Além disso, inclui uma menor proporção de pessoas acima de 60 anos do que os planos individuais/familiares, consequentemente os custos médios com internações, consultas médicas, demais despesas assistenciais e terapias tendem a ser menores nos planos coletivos. Os custos com exames complementares e outros procedimentos ambulatoriais, por sua vez, muitas vezes são solicitados pela própria empresa, o que tende a aumentar a utilização e os gastos com esses itens.

\section{CONSIDERAÇÕES FINAIS}

Esse estudo analisou os impactos do envelhecimento populacional, do aumento na expectativa de vida e seus diferenciais por sexo nos gastos futuros dos planos de saúde privados. Alguns pressupostos foram assumidos: taxa média de cobertura dos planos de saúde dos anos 2014, 2015 e 2016, fixas para os anos de projeção; não há inflação, inovações médico-tecnológicas, entre outros fatores de avanços médicos; e o custo médio por exposto de cada item de despesa assistencial é igual para ambos os sexos e fixo ao longo do tempo.

Os resultados apontam que o processo de envelhecimento populacional acontece de forma mais acentuada na população de beneficiários de planos individuais/familiares de assistência médica, em comparação com as pessoas presentes em planos coletivos. Conforme os resultados das projeções, o topo da pirâmide etária dos planos individuais/familiares será significantemente mais largo do que dos planos coletivos, ressaltando ainda que o alargamento será mais intenso no sexo feminino. Destaca-se que a participação de pessoas adultas (entre os 18 e 50 anos de idade) nos planos coletivos é expressivamente maior que nas demais idades, assim como em comparação com os planos individuais/familiares. Isso porque os planos coletivos são demandados por empresas, conselhos, sindicatos ou associações para oferecer assistência médica e/ou odontológica às pessoas com vinculação institucional, as quais geralmente estão em idade ativa. Considerando a hipótese de Albuquerque e colaboradores ${ }^{24}$, sobre a relação entre a contratação de um plano de saúde com emprego formal e a renda, e os achados sobre crescimento dos planos coletivos em detrimento dos individuais/familiares, entre 2000 e 2006, os resultados deste estudo sugerem que processo de envelhecimento populacional é mais uma ameaça para sustentabilidade futura dos planos individuais/familiares.

As projeções dos custos assistenciais apontam que as internações esboçam os maiores valores monetários absolutos entre os itens assistenciais, tanto para os planos com tipo de contratação individual/familiar, como para os coletivos, em todos os anos projetados. Em segundo lugar, estão os exames complementares. Além desses resultados, observou-se que para ambos os tipos de contratação e todos os custos assistenciais, o valor total despendido com as mulheres é significativamente superior aos despendidos com os homens, como consequência da maior proporção de mulheres nas faixas etárias mais elevadas. Apesar de o estudo ter assumido o mesmo custo médio por exposto de cada item de despesa assistencial para ambos os sexos, é importante reconhecer que as mulheres utilizam mais os serviços ofertados pelos planos de saúde ${ }^{23}$, o que tende a aumentar ainda mais os custos com elas. Essa maior utilização do serviço pelas mulheres corrobora os achados de estudos que apontam que as mulheres tendem a cuidar mais da própria saúde, ao passo que os homens apresentam comportamentos mais arriscados ${ }^{12}$. Um estudo recente, entretanto, buscou analisar se os homens têm a saúde pior do que as mulheres ${ }^{25}$. A questão foi abordada analisando o diferencial por sexo na mortalidade e as diferenças de saúde ao longo do tempo e entre países. Atualmente, a expectativa de vida 
feminina é maior do que a masculina, mas as mulheres têm mais dificuldade em realizar as atividades instrumentais de vida diária. Os autores apontam, ainda, que a falta de consistência dos dados entre os países, uma vez que os resultados não demonstraram uma tendência clara, indicam a complexidade e os desafios enfrentados na determinação das tendências futuras do diferencial por sexo na saúde.

O mutualismo é princípio que norteia os cálculos das operadoras de saúde e prevê que os custos gerados pela utilização do plano sejam divididos entre todos os seus participantes. Alguns grupos apresentam maiores custos do que outros, como é o caso dos idosos e das mulheres, os quais precisam ser diluídos para viabilizar a participação deles. Por exemplo, o idoso geralmente não produz como produzia na idade adulta ou se aposenta, por isso o seu rendimento individual tende a cair e, por outro lado, passa a necessitar de mais cuidados médicos e hospitalares e a utilizar com mais frequência o plano de saúde. Portanto, apesar de o idoso custar mais ao plano, ele não tem capacidade financeira para arcar com uma mensalidade exorbitante, custo este que precisa ser diluído entre as demais idades por meio da colaboração intergeracional.

A faixa etária que apresenta maior crescimento de custo é a 59 anos ou mais, o que reafirma a significativa influência do processo de envelhecimento populacional no mercado de saúde suplementar. O setor privado de saúde já vem passando por períodos de aumentos nas despesas para todas as idades, entretanto, a última faixa etária requer mais atenção, pois as pessoas idosas necessitam de cuidados em saúde mais frequentes, e tais cuidados muitas vezes são mais custosos que os valores vistos nas demais idades. Destaca-se ainda o maior quantitativo de mulheres nesta faixa de idade em relação aos homens. Nesse sentido, as operadoras de saúde precisam se planejar para garantir a sua solvência financeira e a manutenção de cobertura para idosos e mulheres.

Outra consideração que merece atenção diz respeito às dez faixas etárias determinadas pela ANS para precificação dos planos de saúde. Mais especificamente, em relação ao último grupo etário, pois este será o que mais sofrerá alterações com o processo de envelhecimento da população no decorrer do tempo. Assim, as atuais regras de precificação dadas pela ANS poderão dificultar a manutenção do equilíbrio financeiro e atuarial das operadoras de planos de saúde. Logo, fica a cargo da Agência avaliar detalhadamente este método de precificação, no intuito de garantir a solvência das operadoras de planos privados de assistência à saúde e a manutenção de cobertura de diversos grupos, como idosos e mulheres.

Dada as limitações da análise, os resultados ainda apontam para expressivos aumentos no total dos custos em cada item assistencial, como consequência exclusivamente dos processos demográficos. Caso sejam consideradas as demais variáveis que afetam as despesas, os custos tendem a ser ainda maiores. Desta forma, as operadoras de planos de saúde necessitam dar a devida atenção ao efeito do envelhecimento populacional, assim como os demais fatores que venham a influenciar os gastos.

No que diz respeito aos ganhos em longevidade, foi considerada apenas a expectativa de vida a partir dos 60 anos de idade. Os resultados revelaram que o custo esperado em média com cada beneficiário feminino com 60 anos até o fim da vida é superior aos valores observados para os homens, tanto em planos individuais/familiares, quanto em planos coletivos (empresariais e por adesão). Em ambos os sexos, os itens de despesa assistencial consultas médicas, demais despesas assistenciais, internações e terapias, são mais elevados para os planos individuais/familiares do que os coletivos. No que se refere aos exames e outros procedimentos ambulatoriais, esta relação se inverte. Esse resultado pode ser justificado pelo próprio perfil etário de cada tipo de contratação, que determina o custo médio anual de um indivíduo na faixa etária de precificação 59 anos, pois nos planos individuais/familiares há mais idosos com idades mais avançadas e que utilizam com maior frequência serviços como internações, por exemplo. No caso dos planos coletivos, o custo é menor para essa faixa etária de precificação, justamente pela menor presença de idosos com idades mais avançadas. Além disso, como os planos coletivos são destinados ao grupo de pessoas inseridas no mercado de trabalho, os participantes tendem a usar mais os itens exames complementares e outros procedimentos ambulatoriais devido à demanda da própria empresa, como monitoramento da saúde de seus 
trabalhadores. Futuramente, uma análise mais minuciosa do último grupo etário, abrindo estas idades, pode ajudar a elucidar essas questões.

Um outro ponto que não foi considerado nas projeções, mas que pode impactar a sustentabilidade dos planos, é o desemprego. Em momentos de crise, a relação de desemprego e quantidade de beneficiários na saúde suplementar pode impactar a receita e consequentemente o resultado financeiro das operadoras de planos de saúde, principalmente os planos coletivos empresariais.

Portanto, tem-se então a necessidade de enfatizar, mesmo com as limitações dos cenários considerados nas projeções e estimativas, que o processo de envelhecimento e os ganhos em longevidade da população de beneficiários da saúde suplementar apresentam efeitos expressivos nos custos assistenciais. Desta forma, as operadoras de saúde precisam repensar as políticas de cuidados preventivos, a atenção preventiva nos cuidados com a saúde de seus usuários, além de outras ações que visem uma melhor qualidade de vida para estas pessoas e a menor necessidade de utilização de itens assistenciais, como internação e exames complementares, em especial para os idosos.

\section{REFERÊNCIAS}

1. Alves JED. Transição demográfica, transição da estrutura etária e envelhecimento. Revista Longeviver. 2014;40:8-15.

2. Paiva PTA, Wajnman S. From the causes to the economic consequences of the demographic transition in Brazil. Rev Bras Estud Popul. 2005;22(2):303-22.

3. Reis CS. Envelhecimento populacional e gastos com internação do SUS: uma análise realizada para todas as regiões do país entre 2000 e 2010 [dissertação]. Minas Gerais: Universidade Federal de Minas Gerais; 2013.

4. Perez M. A população idosa e o uso de serviços de saúde. Revista HUPE. 2008; 7(1):30-7.

5. Glei DA, Horiuchi S. The narrowing sex differential in life expectancy in high-income populations: effects of differences in the age pattern of mortality. Population studies. 2007;61(2):141-59. http://dx.doi. org/10.1080/00324720701331433. PMid:17558883.

6. Oksuzyan A. Sex differences in health and survival. In: G. Doblhammer, J. Gumà, editors. A demographic perspective on gender, family and health in Europe. Cham: Springer International Publishing;2018. p. 65-100

7. Vallin J, Meslé F. Convergences and divergences in mortality: a new approach to health transition. Rostock: Max Planck Institute for Demographic Research; 2004.

8. Wisser O, Vaupel JW. The sex differential in mortality: a historical comparison of the adult-age pattern of the ratio and the difference. Rostock: Max Planck Institute for Demographic Research; 2014.

9. Luy $M$, Wegner-Siegmundt $C$. The impact of smoking on gender differences in life expectancy: more heterogeneous than often stated. Eur J Public Health. 2015;25(4):706-10. http://dx.doi.org/10.1093/ eurpub/cku211. PMid:25505018.

10. Luy M, Gast K. Do women live longer or do men die earlier? Reflections on the causes of sex differences in life expectancy. Gerontology. 2014;60(2):143-53. http://dx.doi.org/10.1159/000355310. PMid:24296637.

11. Sundberg L, Agahi N, Fritzell J, Fors S. Why is the gender gap in life expectancy decreasing? The impact of age- and cause-specific mortality in Sweden 1997-2014. Int J Public Health. 2018;63(6):673-81. http:// dx.doi.org/10.1007/s00038-018-1097-3. PMid:29654335.

12. Kalben BB. Why men die younger: causes of mortality differences by sex. N Am Actuar J. 2000;4(4):83-111. http://dx.doi.org/10.1080/10920277.2000.10595939.

13. Case A, Paxson C. Sex differences in morbidity and mortality. Demography. 2005;42(2):189-214. http:// dx.doi.org/10.1353/dem.2005.0011. PMid:15986983.

14. Ribas BKC. Processo regulatório em saúde suplementar: dinâmica e aperfeiçoamento da regulação para a produção da saúde [dissertação]. Curitiba: Universidade Federal do Paraná; 2009.

15. Ziroldo RR, GimeneS RO, Castelo C Jr. A importância da saúde suplementar na demanda da prestação dos serviços. O Mundo da Saúde. 2013;37(2):216-21.

16. Instituto Brasileiro de Geografia e Estatística. Projeções da população: Brasil e unidades da federação [internet]. 2013 [citado em 2017 Set 30]. Disponível em: https://www.ibge.gov.br/estatisticas-novoportal/ sociais/populacao/9109-projecao-da-populacao.html. 
17. Agência Nacional de Saúde Suplementar. Painel de Precificação de Planos de Saúde [internet]. 2017. [citado em 2017 Fev 11]. Disponível em: http://www.ans.gov.br/2a-ans-2/3432-painel-de-precificacao-planos-de-saude?highlight=WyJWYWIuZ WwiLCJkZSIsInByZWNpZmljYVx1MDBIN1x1MDBIM28iLCJwYWIuZWwgZGUiLCJwYWluZWwgZGUgcHJIY2lmaWNhXHUwMGU3XHUwMGUzbylsImRIIHByZWNpZmljYVx1MDBIN1x1MDBIM28iXQ==.

18. MillerT. Increasing Longevity and Medicare Expenditures [internet]. 2000 [citado em 2017 Nov 3]. Disponível em: http://citeseerx.ist.psu.edu/viewdoc/download?doi=10.1.1.522.385\&rep=rep1\&type=pdf.

19. Rodrigues CG. Dinâmica demográfica e internações hospitalares: uma visão prospectiva para o Sistema Único de Saúde (SUS) em Minas Gerais, 2007 a 2050 [tese]. Minas Gerais: Universidade Federal de Minas Gerais; 2010.

20. Carneiro LAF. Envelhecimento populacional e os desafios para o sistema de saúde brasileiro [internet] 2013 [citado em 2017 Nov 3]. Disponível em: https://www.ibedess.org.br/imagens/biblioteca/939_ envelhecimentopop2013.pdf.

21. Brasil. Diretoria Colegiada da Agência Nacional de Saúde Suplementar. Resolução Normativa. ANS [internet], Brasília, 22 de dezembro de 2003 [citado em 2017 Maio 11]. Disponível em: http://www.ans. gov.br/component/legislacao/?view=legislacao\&task=PDFAtualizado\&format=raw\&id=NzQ4.

22. Agência Nacional de Saúde Suplementar. Caderno de Informações da Saúde Suplementar: beneficiários, operadoras e planos [Internet]. 2016 [citado em 2017 Ago 12]. Disponível em: http://www.ans.gov. br/images/stories/Materiais_para_pesquisa/Perfil_setor/Caderno_informacao_saude_suplementar/ Caderno_setembro_2016.pdf.

23. Duarte ALCM, Oliveira FM, Santos AA, Santos BFC. Evolução na utilização e nos gastos de uma operadora de saúde. Ciênc saúde colet. 2017;22(8):2753-62.

24. Albuquerque C, Piovesan MF, Santos IS, Martins ACM, Fonseca AL, Sasson D, et al. A situação atual do mercado da saúde suplementar no Brasil e apontamentos para o futuro. Cien Saude Colet. 2008;13(5):142130. http://dx.doi.org/10.1590/S1413-81232008000500008. PMid:18813645.

25. Crimmins EM, Shim H, Zhang YS, Kim JK. Differences between men and women in mortality and the health dimensions of the morbidity process. Clin Chem. 2019;65(1):135-45. http://dx.doi.org/10.1373/ clinchem.2018.288332. PMid:30478135. 